\section{(1) \\ CrossMark}

\title{
COMET: a multicomponent home-based disease-management programme versus routine care in severe COPD
}

\author{
Romain Kessler ${ }^{1}$, Pere Casan-Clara ${ }^{2}$, Dieter Koehler ${ }^{3}$, Silvia Tognella ${ }^{4}$, \\ Jose Luis Viejo ${ }^{5}$, Roberto W. Dal Negro ${ }^{6}$, Salvador Díaz-Lobato ${ }^{7}$, \\ Karina Reissig ${ }^{3}$, José Miguel Rodríguez González-Moro ${ }^{8}$, Gilles Devouassoux ${ }^{9}$, \\ Jean-Michel Chavaillon ${ }^{10}$, Pierre Botrus ${ }^{11}$, Jean-Michel Arnal ${ }^{12}$, \\ Julio Ancochea ${ }^{13}$, Anne Bergeron-Lafaurie ${ }^{14}$, Carlos De Abajo ${ }^{15}$, \\ Winfried J. Randerath ${ }^{16}$, Andreas Bastian ${ }^{17}$, Christian G. Cornelissen ${ }^{18}$, \\ Georg Nilius $^{19}$, Joëlle B. Texereau ${ }^{20,21}$ and Jean Bourbeau ${ }^{22}$
}

@ERSpublications

Lower all-cause mortality in multicomponent home-based disease management programme $v s$ routine care in severe COPD http://ow.ly/sykh30gS5XO

Cite this article as: Kessler R, Casan-Clara P, Koehler D, et al. COMET: a multicomponent home-based disease-management programme versus routine care in severe COPD. Eur Respir J 2018; 51: 1701612 [https://doi.org/10.1183/13993003.01612-2017].

ABSTRACT The COPD Patient Management European Trial (COMET) investigated the efficacy and safety of a home-based COPD disease management intervention for severe COPD patients.

The study was an international open-design clinical trial in COPD patients (forced expiratory volume in $1 \mathrm{~s}<50 \%$ of predicted value) randomised 1:1 to the disease management intervention or to the usual management practices at the study centre. The disease management intervention included a selfmanagement programme, home telemonitoring, care coordination and medical management. The primary end-point was the number of unplanned all-cause hospitalisation days in the intention-to-treat (ITT) population. Secondary end-points included acute care hospitalisation days, BODE (body mass index, airflow obstruction, dyspnoea and exercise) index and exacerbations. Safety end-points included adverse events and deaths.

For the 157 (disease management) and 162 (usual management) patients eligible for ITT analyses, allcause hospitalisation days per year $(\mathrm{mean} \pm \mathrm{SD}$ ) were $17.4 \pm 35.4$ and $22.6 \pm 41.8$, respectively (mean difference $-5.3,95 \% \mathrm{CI}-13.7$ to $-3.1 ; \mathrm{p}=0.16)$. The disease management group had fewer per-protocol acute care hospitalisation days per year $(\mathrm{p}=0.047)$, a lower BODE index $(\mathrm{p}=0.01)$ and a lower mortality rate $(1.9 \%$ versus $14.2 \% ; \mathrm{p}<0.001$ ), with no difference in exacerbation frequency. Patient profiles and hospitalisation practices varied substantially across countries.

The COMET disease management intervention did not significantly reduce unplanned all-cause hospitalisation days, but reduced acute care hospitalisation days and mortality in severe COPD patients.

This article has supplementary material available from erj.ersjournals.com

Received: Aug 072017 | Accepted after revision: Oct 132017

This study is registered at ClinicalTrials.gov with identifier NCT01241526.

Support statement: This study was funded by Air Liquide Healthcare. Funding information for this article has been deposited with the Crossref Funder Registry.

Conflict of interest: Disclosures can be found alongside this article at erj.ersjournals.com

Copyright @ERS 2018 
Affiliations: ${ }^{1}$ Pneumologie, Fédération de Médecine Translationnelle de Strasbourg (FMTS), Université de Strasbourg, Strasbourg, France. ${ }^{2}$ Asturias University Hospital, Oviedo, Spain. ${ }^{3}$ Kloster Grafschaft Specialized Hospital, Schmallenberg, Germany. ${ }^{4}$ Bussolengo General Hospital, Bussolengo, Italy. ${ }^{5}$ Burgos University Hospital, Burgos, Spain. ${ }^{6}$ Bussolengo Hospital, Bussolengo, Italy. ${ }^{7}$ Ramón y Cajal University Hospital, Research Institute IRYCIS, Alcalá de Henares University, Madrid, Spain. ${ }^{8}$ Principe de Asturias University Hospital, Madrid, Spain. ${ }^{9}$ Croix Rousse Hospital, Lyon, France. ${ }^{10}$ Antibes-Juan-les-Pins Hospital Center, Antibes, France. ${ }^{11}$ Metz-Thionville Hospital Center, Thionville, France. ${ }^{12}$ Sainte Musse Hospital, Toulon, France. ${ }^{13}$ Pneumology Dept, La Princesa Hospital, Universidad Autónoma de Madrid, Madrid, Spain. ${ }^{14}$ Pneumology Dept, Saint-Louis Hospital, Paris, France. ${ }^{15}$ Burgos University Hospital, Burgos, Spain. ${ }^{16}$ Dept of Pneumology, Bethanien C.V. Hospital, University of Cologne, Solingen, Germany. ${ }^{17}$ St Mary's Hospital Kassel GmbH, Kassel, Germany. ${ }^{18}$ Section for Pneumology, University Hospital Aachen, Aachen, Germany. ${ }^{19}$ Dept of Pneumology, HELIOS Klinik Hagen-Ambrock, University of Witten/Herdecke, Hagen, Germany. ${ }^{20} \mathrm{AP}-\mathrm{HP}$, Dept of Respiratory Physiology, Cochin Hospital, René Descartes University, Paris, France. ${ }^{21}$ Air Liquide Healthcare, Medical Research and Development, Jouy-en-Josas, France. ${ }^{22}$ FRCP Respiratory Epidemiology and Clinical Research Unit, Research Institute of the McGill University Health Centre, Montréal, QC, Canada.

Correspondence: Jean Bourbeau, Respiratory Epidemiology and Clinical Research Unit (RECRU), Research Institute of the McGill University Health Centre, 5252 de Maisonneuve, 3D.62, Montréal, QC, Canada.

E-mail: jean.bourbeaudamcgill.ca

\section{Introduction}

Chronic obstructive pulmonary disease (COPD) is a treatable condition that substantially impacts daily life and affects over $10 \%$ of adults aged over 40 years [1]. Patients with severe to very severe COPD have worse health status [2], higher risk of hospitalised exacerbations, higher acute care burdens [1,3] and higher mortality rates than patients with mild to moderate disease [4-7]. This was also shown in the TIOSPIR (Tiotropium Safety and Performance in Respimat) trial, where the risks of respiratory-related deaths and hospitalised exacerbations were greater in GOLD (Global Initiative for Obstructive Lung Disease) C or D patients than in GOLD A patients [8]. Better control of exacerbation frequency and severity could slow disease progression and reduce hospitalisation $[9,10]$, especially in patients with severe COPD who encounter difficulties in managing their disease on a daily basis.

COPD patients and their families are obligated to take responsibility for daily care and must bear most of the care burden. Importantly, patients must learn how to cope with their disease, recognise when they need professional care, adopt a healthy lifestyle, and avoid modifiable risk factors for exacerbations and hospitalisations. In trying to fill this care gap, a number of self-management programmes have been studied in clinical trials [11-17]. Although the programmes and outcomes of these studies have varied widely, thus complicating identification of the most effective strategies, important programme considerations include COPD severity, patient comorbidities, and flexibility towards the personal goals of the patient [18]. Effective self-management programmes have focused on patient education, behaviour modification, patient motivation through coaching by a health professional, and prompt access to healthcare when needed $[11,19,20]$.

The COPD Patient Management European Trial (COMET) was an international, randomised, controlled clinical trial used to investigate the effectiveness, safety and cost-effectiveness of a multicomponent home-based COPD disease management intervention specifically adapted for patients with severe COPD versus the usual patient management [21]. The COMET programme was designed to be well integrated, coherent, and carefully tuned to patient needs and disease severity. This was accomplished through an integrated approach of care that included: a standardised self-management programme and coaching with a well-trained case manager; close home monitoring and care coordination through an e-health platform for early detection of exacerbations; and feedback and quality control to optimise delivery of the interventions [22]. It was hypothesised that the home-based disease management intervention would help patients adopt necessary coping behaviours and consequently avoid some hospitalisations, and reduce the length of stay for others.

\section{Methods}

Patients

COPD patients aged $\geqslant 35$ years were eligible to be included if they had: a post-bronchodilator forced expiratory volume in $1 \mathrm{~s}\left(\mathrm{FEV}_{1}\right) /$ forced vital capacity $(\mathrm{FVC})$ ratio $\leqslant 70 \%$; an $\mathrm{FEV}_{1}<50 \%$ of the predicted value; $a \geqslant 10$ pack-year smoking history; and at least one severe exacerbation in the previous year. Patients could receive all relevant COPD treatments, including long-term oxygen therapy (LTOT) and home mechanical ventilation (HMV). Patients were not eligible if, in the opinion of the recruiting investigator, they were: not expected to survive longer than 6 months; unable to read or speak the country language or 
had cognitive/psychiatric disease; on continuous treatment of $>10 \mathrm{mg}$ per day prednisone or equivalent for more than 6 weeks; or living in a nursing home.

\section{Study design}

COMET was a randomised, open-design, parallel-group trial conducted at 33 centres across France (12 centres), Germany (eight), Italy (six) and Spain (seven), and was described in a methods paper that included the study protocol (supplementary material) [21]. Briefly, patients with severe COPD were randomised in a 1:1 fashion, to be managed through routine care and follow-up only (UM group) or to receive the multicomponent home-based COPD disease management intervention (DM group) in addition to routine care and follow-up. Each patient enrolled was assessed for respiratory and global health status during a 3- to 5-week run-in period, and then followed up over a subsequent 12-month period. During follow-up, patients were required to have a hospital visit once every 3 months, and were contacted by the hospital staff by phone between visits to collect healthcare use information using a standardised script. Ethical conduct of the study has been described previously [21]. All patients provided written informed consent. During the study, the protocol was amended to accelerate recruitment and allow study completion (supplementary material).

Patients were allocated to groups in a 1:1 fashion according to a pre-specified randomisation list generated before the study by a partial-minimisation computer algorithm under supervision of the study sponsor. Patients were assigned a randomisation number by study staff at each centre in sequential numerical order through a telephone-based interactive voice response system. Randomisation was stratified by smoking status (current or former), need for respiratory assistance (none, or on LTOT and/or HMV), and centre. For practical reasons, the study was open; neither the patients nor the investigators were blinded to the COPD management strategy.

\section{Multicomponent home-based disease management intervention and routine care}

The home-based disease management intervention in COMET included a self-management programme, home monitoring and an e-health telephone/web platform (figure 1) [21].

The self-management programme was based on the "Living Well with COPD" programme developed at the Montreal Chest Institute of the McGill University Health Centre, Montreal, QC, Canada (www. livingwellwithcopd.com) [13]. Adaptations to the original programme were made to account for greater COPD severity, and oxygen use, and for the specific language, cultural and medical practices of each country. Home monitoring consisted of the transmission of health status information by patients using a telephone-based questionnaire at least once per week, and each day they began to experience symptom worsening. An e-health telephone/web platform allowed timely patient follow-up by case managers for early detection of potential exacerbations and symptom worsening. Information was transmitted to the hospital physician via the web platform to coordinate healthcare and early treatment when necessary. Physicians made all decisions regarding medication to reduce the risk of inappropriate medication.

The UM (control) group received the usual or routine COPD care and patient follow-up practices used at each investigational centre. Site-specific usual management practices (e.g. centre-specific COPD educational booklets or programme information, if any) were collected at the beginning and end of the patient inclusion period.

All patients on LTOT were monitored with a NOWOX medical device (Air Liquide, Paris, France) to record time of daily oxygen use and respiratory rate.

\section{Outcomes}

The primary outcome was the number of unplanned all-cause hospitalisation days, normalised to 1 year of follow-up for each randomised patient. Hospitalisation days included a subsequent stay in a nursing facility if it followed the hospitalisation without a return home. Because hospitalisation decisions might have been subject to local practices, social considerations, bed availability, and so on, all hospitalisations were reviewed by an independent, three-member end-point validation committee (EVC) to assess the reason and appropriateness of each hospitalisation, determine whether the hospitalisation fulfilled the protocol definition, and determine the extent to which it was to be considered in the efficacy assessments (fully, partially, not at all).

Supportive hospitalisation outcomes were the number of unplanned all-cause hospitalisation days in acute care wards (not including days in nursing facilities) for COPD exacerbation; this was confirmed by the EVC.

Secondary outcomes included: the number of COPD exacerbations (mild: symptom worsening; moderate: requiring antibiotic or corticosteroid treatment; severe: resulting in hospitalisation and/or death); the 


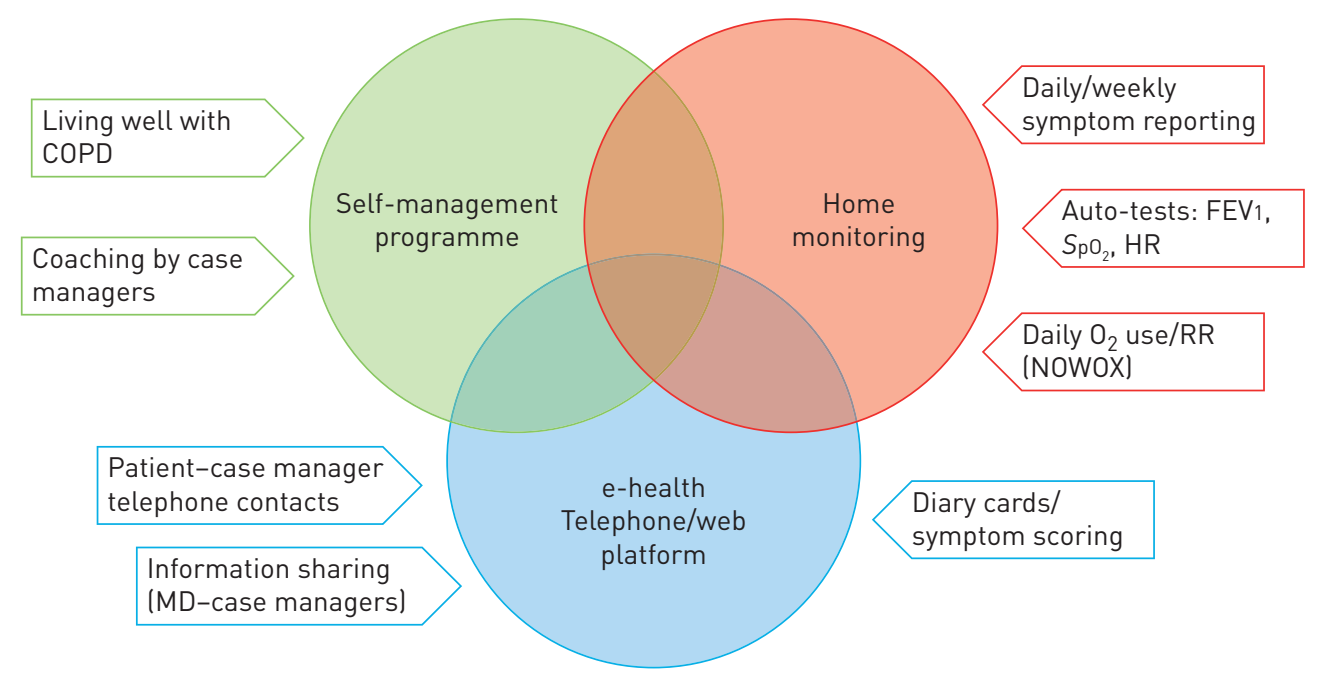

COMET Aims:

Improve patient knowledge, skills, self-management capabilities

Timely detection and treatment of significant clinical worsening

Case manager is first line, physician if required

FIGURE 1 Multicomponent home-based disease management intervention in COMET. In the disease management arm of COMET [21], patients received patient education and coaching based on the "Living Well with COPD" self-management programme (www.livingwellwithcopd.com) [13] from COMET-trained case managers, who were healthcare professionals familiar with home-based interventions and chronic patient care. Key facets of the programme included: 1) patient education and motivation by case managers, with the goal of attaining sustainable self-management skills and behavioural changes; and 2) an action plan to prevent exacerbations, with decision-making and actions to be taken in case symptoms worsen. Patients proactively communicated health status updates whenever their symptoms worsened or at least weekly. Information was relayed automatically to an e-health web platform that registered a status of well-being, worsening, or alarm. For symptom worsening or alarm status, case managers contacted the patient to confirm symptom status. A confirmed alarm status was referred to the investigator for same-day medical assessment and follow-up. Patients also self-monitored forced expiratory volume in $1 \mathrm{~s}$ (FEV 1 ), arterial oxygen saturation measured by pulse oximetry $\left(\mathrm{SpO}_{2}\right)$, and heart rate (HR). For patients on long-term oxygen therapy, daily oxygen use and respiration rate (RR) were recorded by the NOWOX in-line monitoring device. COPD: chronic obstructive pulmonary disease.

6-min walking distance (6MWD); BODE (body mass index, airflow obstruction, dyspnoea and exercise) index and its separate components [23]; anxiety and depression, as assessed by the Hospital Anxiety and Depression Scale (HADS) [24]; and health status, as assessed by the COPD-specific version of the St George's Respiratory Questionnaire (SGRQ-C) [25].

Safety outcomes included adverse events, serious adverse events and deaths.

Medico-economic outcomes included overall costs, cost-effectiveness and cost-utility; their evaluations will be published separately.

\section{Statistical analysis}

The primary analysis of the primary outcome was performed in the intention-to-treat (ITT) population (all patients who entered the follow-up period). The number of hospitalisation days fitting the established criteria was normalised on a yearly basis for each patient and compared between both groups using the non-parametric Wilcoxon rank-sum test.

In supportive analyses, the primary analysis of the primary outcome was repeated in a descriptive and exploratory manner for the overall per-protocol (PP) population and for the ITT and PP populations in each country. A parametric analysis of variance (ANOVA) of the primary outcome was performed on the ITT and PP populations for study group and country effects, and interaction between group and country. The average yearly cumulative duration of unplanned all-cause hospitalisations confirmed by the EVC was also compared between both groups using the Wilcoxon rank-sum test. All other secondary efficacy and safety results were exploratory, exclusively descriptive, and analysed in the ITT population only.

Sample size was calculated based on the primary objective to compare the number of unplanned all-cause hospitalisation days between the two groups. From a previous study, a mean difference of 10 
hospitalisation days per year between the DM group and the UM group, with a common standard deviation of 25 days, was expected [16]. With a two-sided type I error set at $\alpha=0.05$ and power at 0.90 , the minimum sample size was estimated to be 143 evaluable patients per group. With an expected annual dropout rate of $25 \%$, target enrolment for at least the first 3 months of follow-up was 153 patients per group.

\section{Results}

The study was conducted between September 2010 and March 2015. A total of 345 patients were randomised to the DM group $(n=172)$ or the UM group $(n=173)$ (figure 2). Patients were recruited in France $(125 ; 36.2 \%)$, Spain $(94 ; 27.2 \%)$, Germany $(82 ; 23.8 \%)$ and Italy $(44 ; 12.7 \%)$. The DM group included $36.0 \%$ patients from France, $26.2 \%$ from Spain, $24.4 \%$ from Germany, and $13.4 \%$ from Italy. The UM group included 36.4\% patients from France, 28.3\% from Spain, 23.1\% from Germany, and 12.1\% from Italy. In the DM and UM groups, respectively, $15(9 \%)$ and $11(6 \%)$ patients did not complete the initial 5-week run-in period, leaving $157(91 \%)$ and 162 (94\%) patients in the ITT population, $137(80 \%)$ and 128 (74\%) of whom completed at least 12 months of follow-up. Patients were excluded from the PP population (DM group, $\mathrm{n}=134$; UM group, $\mathrm{n}=154$ ) for major protocol deviations, most of which involved

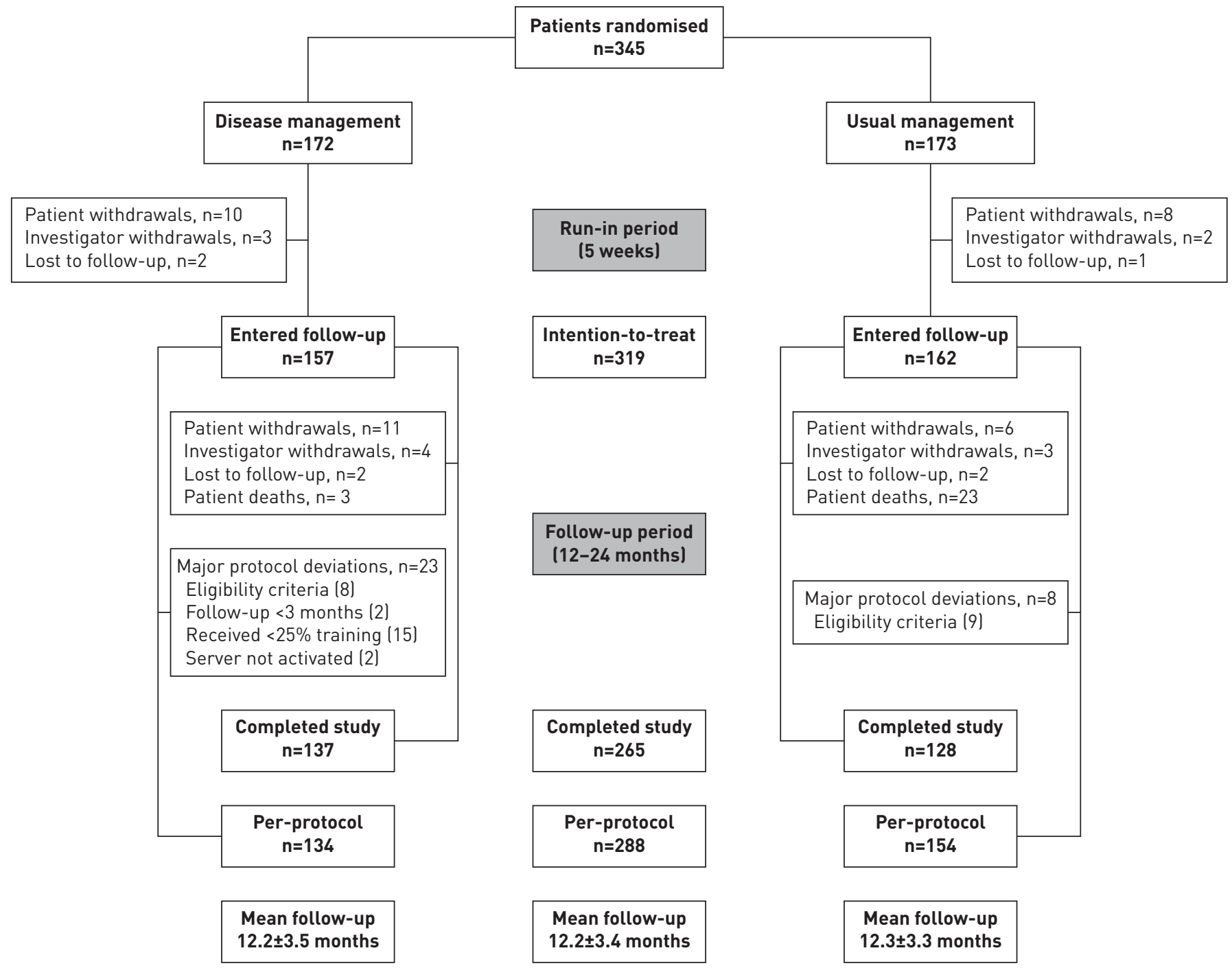

FIGURE 2 Patient flow and disposition. Patients enrolled in the study were randomised in a 1:1 fashion to receive the COMET multicomponent home-based disease management (DM) intervention or the usual management (UM) practised at the centre. Randomised patients received either DM or UM training and education during a 5-week run-in period prior to entering the follow-up period, which was originally set at 2 years but was reduced to 1 year by protocol amendment. Patients who were withdrawn during the run-in period could be replaced by new patients. Patients who entered the follow-up period comprised the intention-to-treat (ITT) population; patients who had no major protocol deviations during the follow-up period comprised the per-protocol population. Mean follow-up for the ITT population was 12.2 months in the DM group and 12.3 months in the UM group. Numerical totals of protocol deviations are shown in parentheses. 
unmet eligibility criteria or involved patients who had received less than $25 \%$ of the planned coaching sessions.

Patient demographics and disease characteristics

Patients in both groups at baseline were similar for most characteristics and notable for their COPD severity (table 1). Patient characteristics varied extensively by country (supplementary table E1).

TABLE 1 Demographics and baseline characteristics of patients in COMET

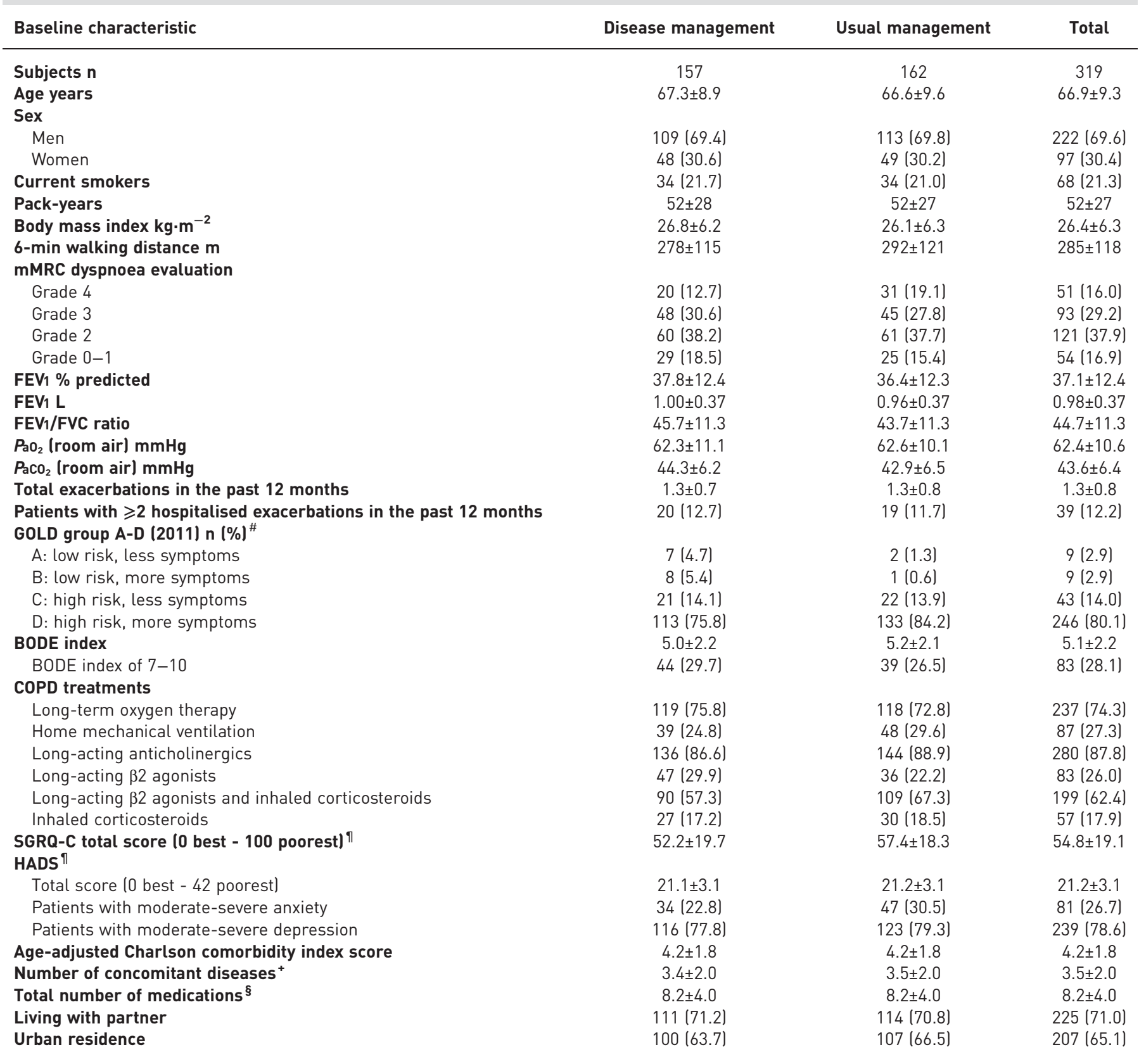

Data are presented as mean \pm SD or $n(\%)$. * : Global Initiative for Chronic Obstructive Lung Disease (GOLD) A-D stage was determined using the modified Medical Research Council (mMRC) dyspnoea scale. ": chronic obstructive pulmonary disease (COPD)-specific version of the Saint George's Respiratory Questionnaire (SGRQ-C) and Hospital Anxiety and Depression Scale (HADS) scores were collected after the run-in period in which patients in the disease management group had already received four home coaching sessions. HADS scores of 11-21 indicate moderate-to-severe anxiety or depression. ${ }^{+}$: in patients with at least one concomitant disease. ${ }^{\S}$ : in patients with at least one concomitant treatment. $\mathrm{FEV} 1$ : forced expiratory volume in $1 \mathrm{~s} ; \mathrm{FVC}$ : forced vital capacity; $\mathrm{PaO}_{2}$ : arterial blood oxygen partial pressure; $P$ aco $\mathrm{C}_{2}$ arterial blood carbon dioxide partial pressure; BODE index: body mass index, airflow obstruction, dyspnoea and exercise index. 
Hospitalisation patterns and primary outcome

The distributions of patients according to unplanned all-cause hospitalisation days were similar for each group (figure 3). Half of the patients had no hospitalisation during follow-up. For those who were hospitalised, the largest groups of patients had between 1-10 and 11-20 unplanned hospitalisation days. The numbers of patients with very long stays ( $>60$ days) were similar in each group. Annual unplanned hospitalisation days (mean $\pm \mathrm{SD}$ ) varied substantially by country, with a low of $9.7 \pm 22.7$ in Spain and a high of $28.7 \pm 40.9$ in Germany (supplementary table E1).

Annual unplanned all-cause hospitalisation days (mean \pm SD) were $17.4 \pm 35.4$ in the DM group and 22.6 \pm 41.8 in the UM group (ITT population), with medians (interquartile ranges) of 0 (0-203) days and 5 (0 -259) days, respectively (figure 4). Differences between groups were not significant in the primary non-parametric analysis $(\mathrm{p}=0.161)$ or in an ANOVA comparison of the mean values adjusted for country differences ( -5.3 days, $95 \% \mathrm{CI}-13.7$ to $3.1 ; \mathrm{p}=0.212$ ).

\section{Supportive hospitalisation outcomes}

Similar results were obtained for EVC-confirmed hospitalisation days (mean \pm sD), with $15.5 \pm 32.9$ in the DM group and $20.8 \pm 38.8$ in the UM group ( $p=0.15$, ITT), and for the primary end-point in the PP population (figure 4). Unplanned all-cause days spent in acute care wards were significantly different in the PP population with a difference in country-adjusted means of -8.3 days (95\% CI -16.4 to -0.1 ; $\mathrm{p}=0.047)$. Hospitalisation days for COPD exacerbations represented less than $50 \%$ of unplanned hospitalisation days for both study groups, with a difference in country-adjusted means of -1.5 days (95\% CI -5.5 to $2.5 ; \mathrm{p}=0.46$, ITT). All-cause hospitalisation days in each study group were higher for patients with LTOT/HMV than for the overall study population, but a statistically significant difference between groups was found only in the non-parametric comparison in the PP population. In a multivariate regression analysis, only the requirement for LTOT $\geqslant 15 \mathrm{~h}$ per day was a significant risk factor for unplanned hospitalisation, with a risk ratio of 2.55 (95\% CI 1.17-5.57; p=0.02).

The total numbers of unplanned hospital admissions were similar for both groups (DM group, n=157; UM group, $n=160$ ), as were the EVC-determined primary causes of these admissions (supplementary table E2). COPD exacerbations (69.1\%), pneumonia (9.5\%) and acute cardiac failure (4.4\%) were the most frequent primary causes of hospital admission overall.

\section{Secondary outcomes}

After 12 months of follow-up, numbers of patients with exacerbations, exacerbation frequencies, and the time to a first exacerbation were similar for both groups (table 2). The BODE index improved in $42.4 \%$ of the patients in the DM group versus $30.6 \%$ in the UM group. The baseline-adjusted mean BODE score was significantly lower in the DM group, with a difference of -0.5 ( $95 \%$ CI -0.9 to $-0.1 ; \mathrm{p}=0.01)$. Groups

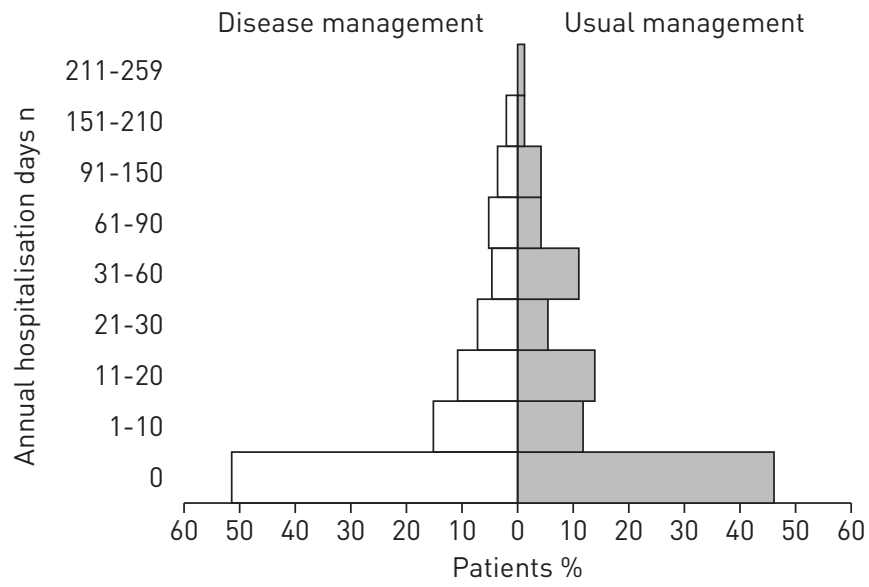

FIGURE 3 Distribution of annualised number of unplanned all-cause hospitalisation days. Patients in each group in the intention-to-treat population were classified by the number of unplanned all-cause hospitalisation days they reported per year. Approximately $50 \%$ of the patients in each group had no unplanned hospitalisation days during the study. Similar numbers of patients in each group had very long hospital stays, with three patients in the home-based disease management group and two in the usual management in range of 151-210 days, and no disease management group patients and two usual management group patients in the range of 211-259 days. 


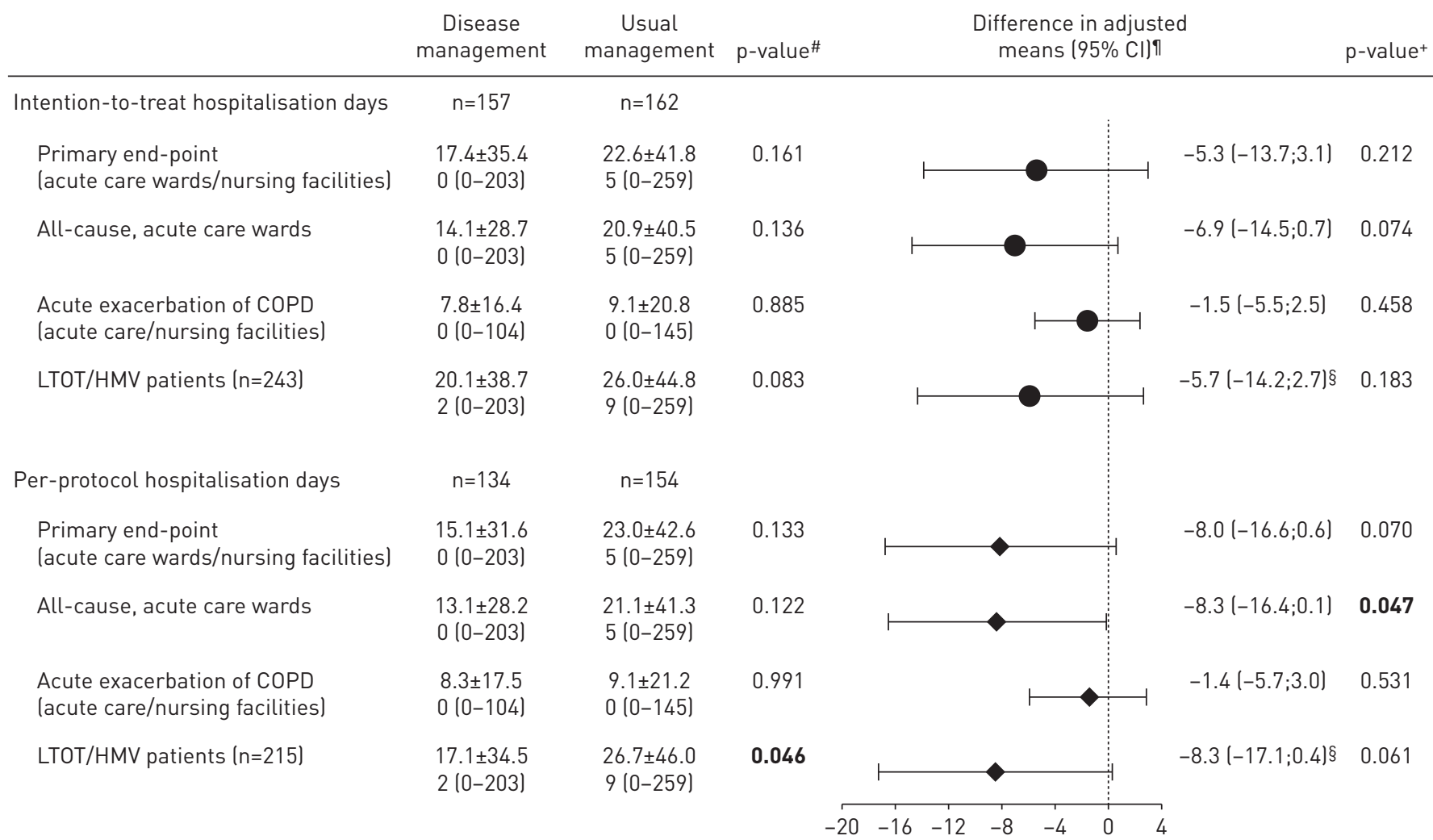

FIGURE 4 Comparison of annual unplanned hospitalisation days in each group by subcategory and population. The annual mean and median number of unplanned all-cause hospitalisation days spent in either acute care wards or nursing facilities are shown for each group in intention-to-treat and per-protocol. Also shown are the annual mean and median numbers of unplanned all-cause hospitalisation days spent in acute care wards only; due to an acute exacerbation of chronic obstructive pulmonary disease (COPD); and for patients on home oxygen therapy and/or home mechanical ventilation (LTOT/HMV). p-values in bold type indicate statistically significant differences. Data are presented as mean \pm SD, median (interquartile range), with difference in adjusted means presented with $95 \%$ confidence intervals. ${ }^{*}$ : non-parametric Wilcoxon rank-sum test; ": means, except for LTOT/HMV, were adjusted for country differences and compared by ANOVA with average yearly number of unplanned hospital days as the response variable; ${ }^{+}$: ANOVA; ${ }^{\S}$ : the means for LTOT/HMV patients were adjusted for the need for respiratory assistance and compared by ANOVA with average yearly number of unplanned hospital days as the response variable.

did not differ significantly for the other secondary outcomes of 6MWD, SGRQ-C or HADS. More patients in the DM group stopped smoking during the study $(\mathrm{p}=0.02)$ and average daily use of LTOT was similar.

\section{Safety outcomes}

Similar proportions of patients in each group experienced at least one adverse event ( $90 \%)$, most of which were COPD exacerbations (table 3). Approximately $60 \%$ of the patients in each group experienced at least one severe adverse event, the most frequent being COPD exacerbation, pneumonia, and acute cardiac or respiratory failure.

A total of 27 patients died during the study, one of which died prior to randomisation. The mortality rate was significantly lower in the DM group (three patients, $1.9 \%$ ) than in the UM group (23 patients, 14.2\%; $\mathrm{p}<0.001$; table 3 and figure 5). The three deaths in the DM group were due to respiratory failure, cardio-respiratory arrest and traumatic pneumothorax. More than half of the deaths (13) in the UM group were attributed to COPD exacerbation; no other cause accounted for more than two deaths. Notable baseline characteristics of the patients who died (determined post hoc) were a low FEV1 (35\% of predicted), GOLD D category (84.6\%), LTOT (84.6\%), poor health status (mean SGRQ-C score of 63.7) and multiple concomitant diseases (mean number of 4.3) (supplementary table E4). These patients also accumulated high numbers of unplanned hospitalisation days during the study (median of 68.0 days). In a post hoc multivariate regression analysis, baseline BODE index $(\mathrm{p}=0.03)$ and the total number of hospitalisation days during the study $(\mathrm{p}<0.001)$ were significant independent predictive factors of death.

\section{COMET disease management intervention and skills acquisition}

Overall, $100 \%$ of the patients in the DM group performed all four initial individual home coaching sessions during run-in, $66.7 \%$ of patients achieved at least $80 \%$ of their planned phone and group coaching 


\section{TABLE 2 Secondary outcomes}

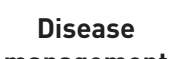

management

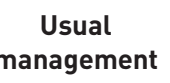

management

\section{Difference in \\ adjusted}

means $(95 \% \mathrm{CI})^{\pi}$

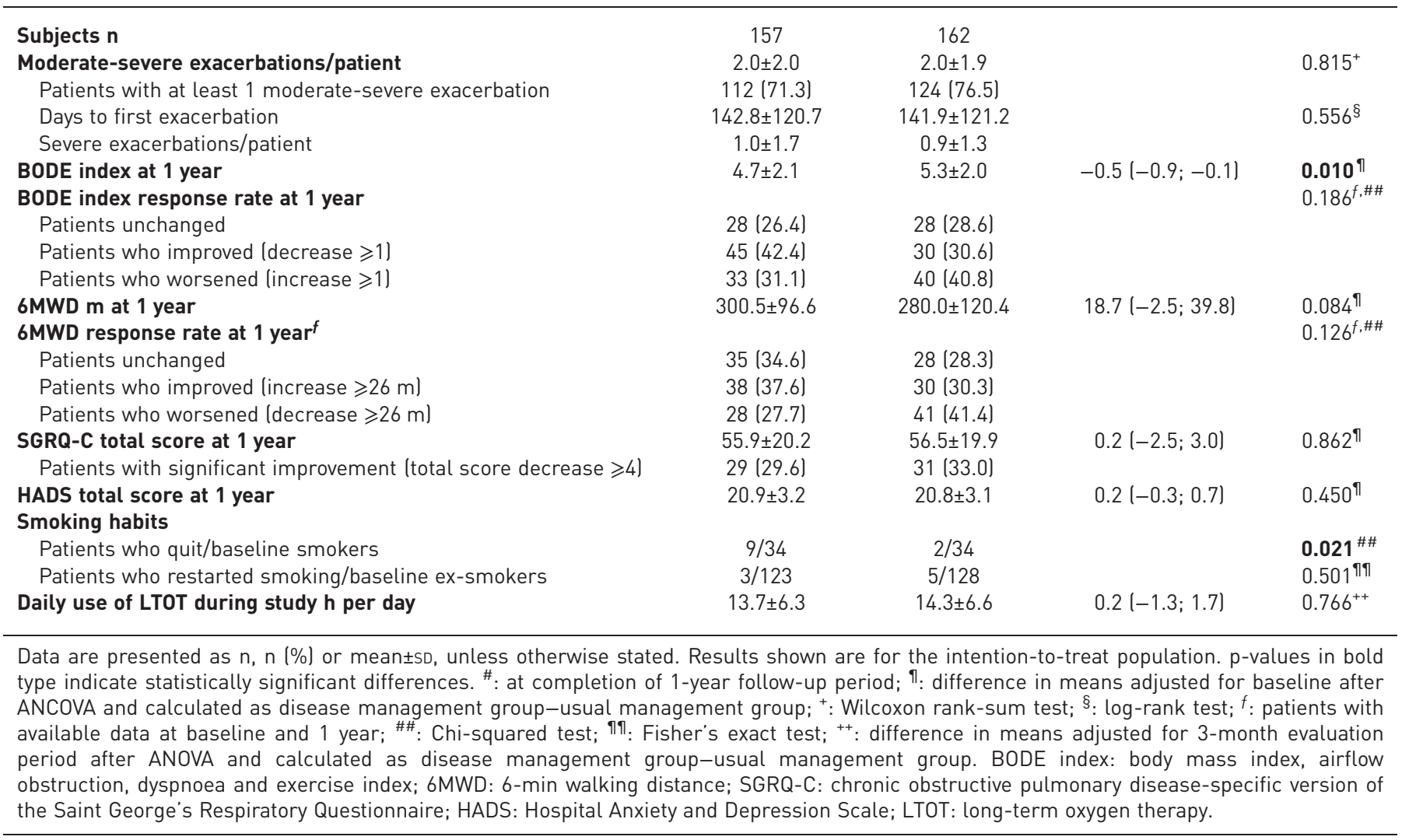

sessions, and $89.7 \%$ achieved at least $80 \%$ compliance for weekly telephone health status transmissions. Mastery of 28 management skills (e.g. inhalation techniques, cough techniques, pursed lip breathing and body positioning, identification of actions to be performed when feeling worse) improved between the first evaluation (eight skills fully mastered by $>75 \%$ of the patients) and the last evaluation (24 skills fully mastered by $>80 \%$ of the patients).

\section{Centre COPD management routine practices}

Centre practices, in terms of the COPD educational programmes routinely implemented in both groups, independent from the COMET disease management intervention, varied widely by country and centre (supplementary table E3). Overall, $22.6 \%$ of the centres provided patients with educational booklets, $18.8 \%$ offered COPD educational sessions, and $40.6 \%$ offered exercise programmes.

\section{Discussion}

A novel multicomponent home-based COPD disease management intervention across four European countries did not achieve a statistically significant difference in the predefined primary outcome, although patients in the DM group had 23\% fewer all-cause hospitalisation days than patients in the UM group. Acute care hospitalisation days in the PP population, BODE index scores at 12 months, and the overall mortality rate, were all statistically significantly lower in the DM group than in the UM group. However, as these were supportive or secondary outcomes intended to support the interpretation of the primary outcome, and mortality was a safety outcome, these results should be considered with caution.

To date, the results of COPD self-management interventions in clinical trials have been inconsistent [26], which has created ambiguity regarding self-management interventions and has discouraged their implementation in clinical practice. Recently, a group of experts reached a consensus regarding a conceptual definition of COPD self-management: “A COPD self-management intervention is structured but personalised and often multi-component, with goals of motivating, engaging and supporting the 
TABLE 3 Safety outcomes: adverse events and mortality

\begin{tabular}{|c|c|c|}
\hline Outcome & $\begin{array}{c}\text { Disease } \\
\text { management }\end{array}$ & $\begin{array}{l}\text { Usual } \\
\text { management }\end{array}$ \\
\hline Subjects $\mathrm{n}$ & 157 & 162 \\
\hline Patients with at least one adverse event & $139(88.5)$ & $151(93.2)$ \\
\hline Total number of adverse events & 683 & 801 \\
\hline \multicolumn{3}{|l|}{$\begin{array}{l}\text { Patients with adverse events that occurred in } \geqslant 3 \% \text { of patients } \\
\text { overall during follow-up }\end{array}$} \\
\hline COPD exacerbation (any severity) & $113(72.0)$ & $124(76.5)$ \\
\hline Pneumonia & $13(8.3)$ & $13(8.0)$ \\
\hline Bronchitis & $10(6.4)$ & $9(5.6)$ \\
\hline Nasopharyngitis & $8(5.1)$ & $5(3.1)$ \\
\hline Dyspnoea & $4(2.5)$ & $6(3.7)$ \\
\hline Acute cardiac failure & $4(2.5)$ & $9(5.6)$ \\
\hline Back pain & $6(3.8)$ & $6(3.7)$ \\
\hline Peripheral oedema & $3(1.9)$ & 7 (4.3) \\
\hline Patients with at least one SAE & $92(58.6)$ & $101(62.3)$ \\
\hline Total number of SAEs & 267 & 312 \\
\hline \multicolumn{3}{|l|}{$\begin{array}{l}\text { Patients with SAEs that occurred in } \geqslant 2 \% \text { of patients overall } \\
\text { during follow-up }\end{array}$} \\
\hline COPD exacerbation & $67(42.7)$ & $78(48.1)$ \\
\hline Pneumonia & 9 (5.7) & $10(6.2)$ \\
\hline Acute cardiac failure & $3(1.9)$ & $7(4.3)$ \\
\hline Acute respiratory failure & $4(2.5)$ & $3(1.9)$ \\
\hline Deaths during follow-up & $3(1.9)$ & $23(14.2)^{\pi}$ \\
\hline \multicolumn{3}{|l|}{ Fatal SAEs during follow-up } \\
\hline COPD exacerbation & $0(0.0)$ & $13(8.0)$ \\
\hline Pneumonia & $0(0.0)$ & $2(1.2)$ \\
\hline Acute respiratory failure & $0(0.0)$ & $1(0.6)$ \\
\hline Respiratory failure & $1(0.6)$ & $0(0.0)$ \\
\hline Hypercapnic coma & $0(0.0)$ & $2(1.2)$ \\
\hline Hypoxic-ischaemic encephalopathy & $0(0.0)$ & $1(0.6)$ \\
\hline Cardio-respiratory arrest & $1(0.6)$ & $1(0.6)$ \\
\hline Bile duct cancer & $0(0.0)$ & $1(0.6)$ \\
\hline Cholangiocarcinoma & $0(0.0)$ & $1(0.6)$ \\
\hline Multi-organ failure & $0(0.0)$ & $1(0.6)$ \\
\hline Biliary tract infection & $0(0.0)$ & $1(0.6)$ \\
\hline Pneumothorax traumatic & $1(0.6)$ & $0(0.0)$ \\
\hline Septic shock & $0(0.0)$ & $1(0.6)$ \\
\hline $\begin{array}{l}\text { Patients who discontinued because of an adverse event during } \\
\text { follow-up }{ }^{\#}\end{array}$ & $4(2.5)$ & $23(14.2)$ \\
\hline
\end{tabular}

Data are presented as $\mathrm{n}(\%)$. Results shown are for the intention-to-treat population. * : one patient was withdrawn for worsening depressive syndrome (non-fatal); १: $p<0.001$ (Chi-squared test). COPD: chronic obstructive pulmonary disease; SAE: serious adverse event.

patients to positively adapt their health behaviour(s) and develop skills to better manage their disease" [19]. In retrospect, COMET nevertheless applied many of these concepts and included quality control to optimise delivery of the self-management component. Two-thirds to $90 \%$ of the DM group patients received at least $80 \%$ of the planned number of coaching sessions, or were $80 \%$ compliant with the weekly phone calls to transmit health status, and most acquired disease management skills. Training and education of COPD patients is necessary but insufficient, and behaviour must be modified successfully to realise tangible benefits $[22,26]$.

Although there appeared to be a study-group effect in many supportive hospitalisation outcomes, a number of factors likely contributed to the lack of statistical significance. Approximately one-half of the patients had no hospitalisations and less than half of the hospitalisation days were due to COPD exacerbation, both of which were unexpected given the high COPD severity and the high proportion of patients on LTOT and/or HMV. Hospitalisations due to COPD exacerbations were slightly higher in the DM group, which could be explained by the slightly, but not significantly, higher mean number of severe exacerbations in the DM group. Statistical power was based on a difference of 10 unplanned hospitalisation days between groups with a standard deviation of 25 days; however, the actual difference 


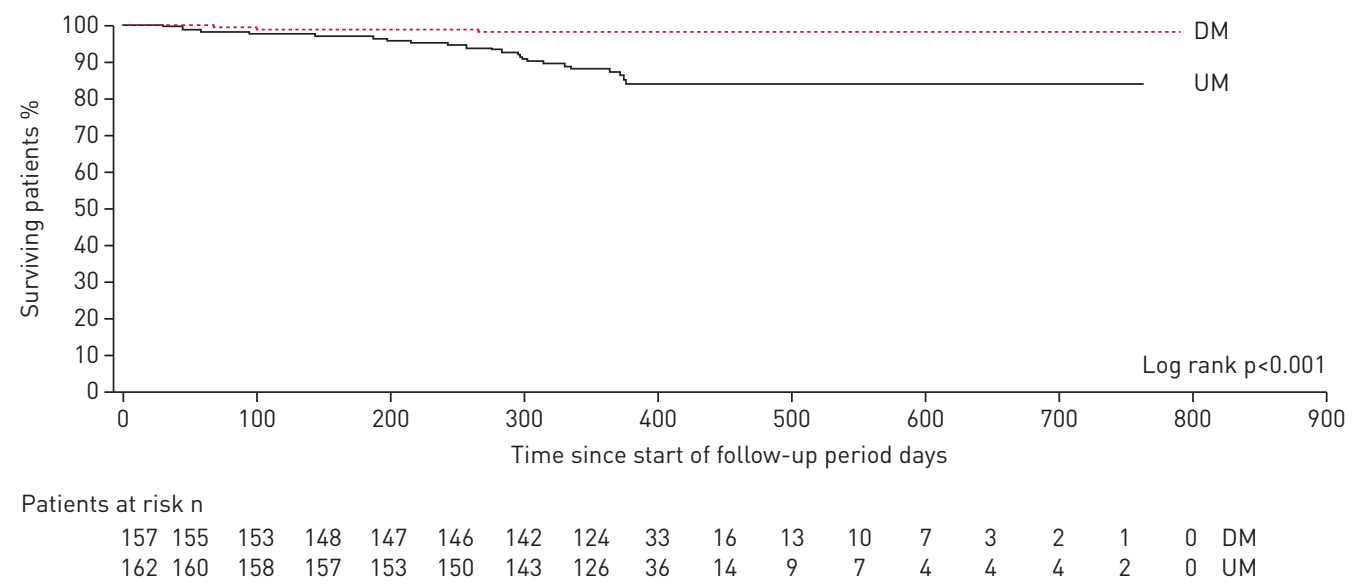

FIGURE 5 Kaplan-Meier analysis of patient mortality. Overall cumulative patient survival rates are shown for the intention-to-treat population for each day of the study. The numbers of patients at risk in each group are shown for each 50 days of the study. During the study, and after 72 patients had already been enrolled, the follow-up period was reduced by protocol amendment from 2 years to 1 year. Thus, some patients were followed for more than 1 year. A total of three patients (1.9\%) in the disease management (DM) group and 23 patients $(14.2 \%)$ in the usual management (UM) group died during the study $(p<0.001)$, mostly owing to respiratory failure (16 deaths). Mean \pm SD time to death in the study was $236 \pm 111$ days.

was only 5.2 days, with standard deviations $>35$ days. This was probably because hospitalisation rates and hospitalisation days differed widely across the four countries owing to country-specific differences in patient baseline characteristics, hospitalisation practices, admission criteria, resource and bed availability, and healthcare reimbursement programmes. For example, the countries differ substantially in hospital bed availability, which is lowest in Spain (297 beds/100000 inhabitants) and highest in Germany (823 beds/ 100000 inhabitants) [27]. This also correlated roughly with hospitalisation days per year (supplementary table E1). It is also possible that we overestimated the hospitalisation risk of this patient population and that a statistically significant difference between groups could have been realised had we enrolled patients with a higher risk of hospitalisation, owing either to comorbidities or to greater COPD severity. Nevertheless, as a consequence of this unforeseen low hospitalisation rate, COMET was statistically underpowered to detect a significant difference in unplanned hospitalisation days, and a larger sample size would have been necessary to compensate for such variability across countries. These factors should be carefully considered in designing future studies of COPD interventions.

The 12-month mortality rate was significantly and markedly lower in the DM group (1.9\%) than in the UM group (14.2\%), the latter of which is consistent with, or lower than, the 12-month mortality rates of $12.5 \%$ [28], 16.8\% [29], 21\% [30] and $>23 \%$ [31] that have been reported previously for COPD patients following a hospitalisation for an acute exacerbation of COPD. That mortality was lower than expected in the DM group, rather than excessively high in the UM group, suggests that the disease management intervention reduced mortality. This may be because most of the deaths (56\%) in the UM control arm were due to COPD exacerbations. It is possible that the disease management intervention reduced mortality by successfully optimising the self-management of exacerbations, leading to early and prompt treatment, which could have prevented additional complications, including death. The home monitoring aspect of the intervention may have also contributed to the success of the intervention by providing a means of rapidly communicating symptoms and disease severity variables to case managers, which may have shortened the time from the beginning of an exacerbation to the institution of appropriate therapy.

Mortality was carefully monitored as a safety outcome in COMET because the disease management intervention was implemented in patients with severe COPD, many of whom had multiple comorbidities and were at high risk of death. Indeed, a prior study by FAN et al. [15] was stopped owing to excess mortality in the self-management group. The COMET disease management intervention was substantively different than that used by FAN et al. [15]. COMET focused on: close patient-physician communication; prompt recognition of exacerbations by regular transmission of symptoms through an e-health system; thorough training and monitoring of case managers, who were followed for quality improvement for the duration of the study; and physicians who maintained responsibility for all medication decisions [21]. By contrast, and as a consequence of the study being conducted at US Veterans Administration hospitals, where healthcare resources may be limited, patients in the FAN et al. [15] study were responsible for much of their own care decisions. Although patients were instructed to call in if they needed to start medications 
for exacerbations, calls were made by only 4.5\%. Furthermore, assessment of exacerbation was done by professionals who may not be experienced in managing this type of patient.

All study deaths in COMET were closely reviewed by the sponsor and the EVC. Although such a large apparent difference in mortality between study groups could have warranted interruption of the study, such a large final reduction in mortality was not expected in COMET, especially since the mortality rate in the usual management arm was not excessive. Additionally, many of the patients who died did so near the end of their first year of follow-up, when most patients had nearly completed the study. Patients who died tended to have a high BODE index and to record high numbers of hospitalisation days during the study, both of which are recognised predictors of mortality in COPD patients [32-34].

The strengths of this study included the use and adaptation of a self-management programme previously demonstrated to be clinically beneficial rather than a new programme. The COMET disease management programme may thus provide a basis for future self-management interventions or for designing studies to evaluate such interventions. Particular attention was given to patient coaching by healthcare professionals (case managers) aimed at optimising self-management through a 4-day training that included a specific focus on motivational communication, continuous access to programme resources (programme objectives, interventions, suggested questions, expected results), and feedback achieved through monthly telephone contacts with a pneumologist from the coordinating study centre in each country. Novel aspects of the disease management intervention included an e-health platform for reporting frequent health status updates, rapid intervention when necessary, and oxygen therapy monitoring. Another strength was the international design of COMET, which was the first study to specifically address a disease management intervention for severe COPD, delivered by centrally trained and supported case managers, across four countries with different medical practices and healthcare systems. Lastly, although the trial was open-designed, hospitalisations were rigorously and blindly reviewed by the EVC and followed-up with additional enquiries if necessary, ensuring the reliability of the outcomes.

The study has some limitations. The inclusion of days spent in acute care wards, as well as subsequent stays in nursing facilities in the primary outcome, may have been suboptimal. Stays in nursing facilities are sometimes extended beyond a clear medical need for hospitalisation, which might have influenced the results. However, the EVC reviewed all stays longer than 30 days and these were removed from the EVC analysis, if warranted. Additionally, the two groups contained similar numbers of patients with very long stays (figure 3). Acute care hospitalisations alone might be considered more appropriate and less subjective, better reflecting immediate patient needs than resource availability or local reimbursement practices. Indeed, acute care hospitalisations appeared to be a more sensitive indicator of hospitalisation needs in COMET, but as the study was not specifically designed to measure this outcome as the primary end-point, these results have limited value. Despite case managers receiving formal training prior to study initiation and being supported throughout the study as part of the quality assurance, there was no quantifiable measure to confirm the quality of intervention delivery. However, the quality assurance used in the study was considered realistic and practical for a real-life disease management programme. Despite the use of three stratification factors to promote the allocation of balanced study groups, differences among countries in baseline characteristics and hospitalisation days were not avoided. These factors, coupled with the site- and country-specific differences in routine care, may have reduced the likelihood of detecting significant differences in many study outcomes. The open design of the study, though necessary owing to the extensive differences between the two disease management programmes, may have led to some bias because doctors could have had a tendency to discharge patients in the disease management arm earlier than those in the usual management arm.

In conclusion, although unplanned annual hospitalisation days tended to be lower in the DM group than in the UM group, the reduction in hospitalisation was statistically significant only for acute care hospitalisation days in patients who received at least $25 \%$ of their planned coaching sessions. One apparent benefit of the disease management intervention was a marked and statistically significant reduction in mortality in which most deaths in the usual management control group resulted from acute exacerbations. The disease management programme may have promoted earlier appropriate treatment for exacerbations, which could have prevented some fatal complications. However, this result should be considered informational because mortality was a safety outcome rather than a primary outcome. Forthcoming medical-economic analyses may also reveal the cost-effectiveness and cost-utility benefits of the disease management intervention. This international study supports the feasibility of a multicomponent home-based disease management intervention in severe COPD patients (many being on LTOT), and demonstrated that most patients adhered to the intervention. However, patient needs and self-management skills at programme entry could be more carefully assessed and followed over time for subsequent programme tailoring. Some very severe patients presenting with severe comorbidities should be clearly identified as they may not be able to fully implement the programme and thus benefit from it. Lastly, the 
characteristics of the healthcare systems and healthcare coordination need to be carefully considered in studies of multicomponent disease management interventions.

\section{Acknowledgements}

Medical writing assistance in the preparation of this manuscript was provided by Kurt Liittschwager (4Clinics, France). Support for this assistance was provided by Air Liquide Healthcare. The authors are also indebted to healthcare professionals and personnel of the home healthcare provider VitalAire in France, Germany, Italy and Spain who were involved in the programme development and implementation, and who generously shared their experience and feedback.

Contributors: J. Bourbeau (Research Institute of the McGill University Health Centre, Montréal, Canada), B. Celli (St. Elizabeth's Medical Center, Boston, USA), J. Rocca (Hospital Clinic, Barcelona, Spain), J. Wedzicha (Royal Free Hospital, London, UK) and B. Housset (Centre Hospitalier Intercommunal de Créteil, Créteil, France) served on the Initial Study Design Expert Committee. C. Bucknall (Glasgow Royal Infirmary, Milngavie, UK), P.R. Burgel (Cochin Hospital, Paris, France) and C. Vogelmeier (Marburg University Hospital, Marburg, Germany) served on the Endpoint Validation Committee. The following individuals contributed to the study at their respective investigational sites. France: M. Canuet, T. Degot, S. Hirschi Santelmo and A. Schuller, Strasbourg Civil Hospital, Strasbourg; D.A. Charpin, N. Lesavre, and A. Palot, North Hospital, Marseille; C. Chenivesse, J. Gonzalez and T. Similowski, Pitié-Salpétrière Hospital Group, Paris; D. Demory and A. Garnero, Sainte Musse Hospital, Toulon; F. Goupil, O. Molinier and A. Paris, Le Mans Hospital Center, Le Mans; J.L. Jagot, M. Lafay and S. Salmeron, Saint-Joseph Hospital Group, Paris; D. Laugros, Beauregard Hospital, Thionville; L. Lerousseau, Antibes-Juan-les-pins Hospital Center, Antibes; G. Lorillon and F. Schlemmer, Saint-Louis Hospital, Paris; I. Olaru, N. Paillot, F. Renolleau and E. Voicu, Metz-Thionville Regional Hospital Center, Metz; J.L. Pepin and R. Tamisier, Grenoble University Hospital Center, Grenoble. Germany: F. Bornitz and F. Herth, Heidelberg Thorax Clinic Gmbh, Heidelberg; M. Dreher, S. Krüger, S. Kweider and T. Müller, University Hospital Aachen; S. Fleimisch, P. Haidl and D.C. Heyse, Kloster Grafschaft Specialized Hospital, Schmallenberg; K.J. Franke, HELIOS Klinik Hagen-Ambrock, University of Witten/Herdecke, Hagen; C. Kropf, University Clinic Ulm, Ulm; D.U. Lange and M. Westhoff, Hemer Pulmonary Clinic, Hemer; C. Priegnitz, Bethanien C.V. Hospital, Solingen; S. Verlaan, St Mary Hospital Kassel GmbH, Kassel. Italy: S. Amaducci, S. Colato, A. Pietra and M. Villani, San Carlo Borromeo Hospital, Milan; S. BErtacco, F. Trevisan and L. Bonadiman, Bussolengo General Hospital, Bussolengo; S. Guarnaccia and P. Scavalli, Andosilla di Civita Castellana Hospital, Civita Castellana; G.P. Mattioli, A. Re and A. Tubaldi, Macerata General Hospital, Macerata; E. Micallef and J. Rolo, Niguarda Hospital Group, Milan; C. Motta and C. Rampoldi, Desio \& Vimercate Hospital Group, Seregno. Spain: C. Aguar Benito, B. Brotons and F. Sanchez-Toril, Arnau de Vilanova Hospital, Valencia; F. Caballero, P. De Lucas and L. Morán, Gregorio Marañón Hospital, Madrid; R. Gallego Dominguez and J.A. Riesco, San Pedro de Alcantara Hospital, Caceres; A. Garcia Garcia and M. Iscar Urrutia, Asturias University Hospital, Oviedo; R.M. Gómez Punter and E. Vázquez Espinosa, La Princesa Hospital, Madrid; M.L. Martinez and T. Pena Miguel, Burgos University Hospital, Burgos; S. Mayoralas and J.L. García González, Ramón y Cajal University Hospital, Madrid.

\section{References}

1 Mannino DM, Buist AS. Global burden of COPD: risk factors, prevalence, and future trends. Lancet 2007; 370: 765-773.

2 Stahl E, Lindberg A, Jansson SA, et al. Health-related quality of life is related to COPD disease severity. Health Qual Life Outcomes 2005; 3: 56.

3 Jansson SA, Andersson F, Borg S, et al. Costs of COPD in Sweden according to disease severity. Chest 2002; 122: 1994-2002.

4 Hilleman DE, Dewan N, Malesker M, et al. Pharmacoeconomic evaluation of COPD. Chest 2000; 118: 1278-1285.

5 Miravitlles M, Murio C, Guerrero T, et al. Costs of chronic bronchitis and COPD: a 1-year follow-up study. Chest 2003; 123: 784-791.

6 Spencer S, Calverley PM, Burge PS, et al. Impact of preventing exacerbations on deterioration of health status in COPD. Eur Respir J 2004; 23: 698-702.

$7 \quad$ Sullivan SD, Ramsey SD, Lee TA. The economic burden of COPD. Chest 2000; 117: Suppl. 2, 5S-9S.

8 Dusser D, Wise RA, Dahl R, et al. Differences in outcomes between GOLD groups in patients with COPD in the TIOSPIR((R)) trial. Int J Chron Obstruct Pulmon Dis 2016; 11: 133-145.

9 Seemungal TA, Donaldson GC, Bhowmik A, et al. Time course and recovery of exacerbations in patients with chronic obstructive pulmonary disease. Am J Respir Crit Care Med 2000; 161: 1608-1613.

10 Wilkinson TM, Donaldson GC, Hurst JR, et al. Early therapy improves outcomes of exacerbations of chronic obstructive pulmonary disease. Am J Respir Crit Care Med 2004; 169: 1298-1303.

11 Adams SG, Smith PK, Allan PF, et al. Systematic review of the chronic care model in chronic obstructive pulmonary disease prevention and management. Arch Intern Med 2007; 167: 551-561.

12 Bischoff EW, Akkermans R, Bourbeau J, et al. Comprehensive self management and routine monitoring in chronic obstructive pulmonary disease patients in general practice: randomised controlled trial. BMJ 2012; 345 : e7642.

13 Bourbeau J, Julien M, Maltais F, et al. Reduction of hospital utilization in patients with chronic obstructive pulmonary disease: a disease-specific self-management intervention. Arch Intern Med 2003; 163: 585-591.

14 Bucknall CE, Miller G, Lloyd SM, et al. Glasgow supported self-management trial (GSuST) for patients with moderate to severe COPD: randomised controlled trial. BMJ 2012; 344: e1060.

15 Fan VS, Gaziano JM, Lew R, et al. A comprehensive care management program to prevent chronic obstructive pulmonary disease hospitalizations: a randomized, controlled trial. Ann Intern Med 2012; 156: 673-683.

16 Farrero E, Escarrabill J, Prats E, et al. Impact of a hospital-based home-care program on the management of COPD patients receiving long-term oxygen therapy. Chest 2001; 119: 364-369.

17 Rice KL, Dewan N, Bloomfield HE, et al. Disease management program for chronic obstructive pulmonary disease: a randomized controlled trial. Am J Respir Crit Care Med 2010; 182: 890-896. 
18 Effing T, Monninkhof EM, van der Valk PD, et al. Self-management education for patients with chronic obstructive pulmonary disease. Cochrane Database Syst Rev 2007; CD002990.

19 Effing TW, Vercoulen JH, Bourbeau J, et al. Definition of a COPD self-management intervention: International Expert Group consensus. Eur Respir J 2016; 48: 46-54.

20 Global Initiative for Chronic Obstructive Lung Disease (GOLD). Global Strategy for the Diagnosis, Management and Prevention of COPD, 2017. Available from http://goldcopd.org

21 Bourbeau J, Casan P, Tognella S, et al. An international randomized study of a home-based self-management program for severe COPD: the COMET. Int J Chron Obstruct Pulmon Dis 2016; 11: 1447-1451.

22 Bourbeau J, Lavoie KL, Sedeno M. Comprehensive self-management strategies. Semin Respir Crit Care Med 2015; 36: 630-638.

23 Glaab T, Vogelmeier C, Buhl R. Outcome measures in chronic obstructive pulmonary disease (COPD): strengths and limitations. Respir Res 2010; 11: 79.

24 Zigmond AS, Snaith RP. The hospital anxiety and depression scale. Acta Psychiatr Scand 1983; 67: 361-370.

25 Meguro M, Barley EA, Spencer S, et al. Development and validation of an improved, COPD-specific version of the St. George Respiratory Questionnaire. Chest 2007; 132: 456-463.

26 Nici L, Bontly TD, Zuwallack R, et al. Self-management in chronic obstructive pulmonary disease. Time for a paradigm shift? Ann Am Thorac Soc 2014; 11: 101-107.

27 European Commission: Eurostat. Hospital beds by type of care, 2014. 2017 [cited 2017 October]; http://ec.europa. eu/eurostat/statistics-explained/index.php/File:Hospital_beds_by_type_of_care,_2014.png Last accessed: October 2017.

28 Mekov E, Slavova Y, Tsakova A, et al. One-year mortality after severe COPD exacerbation in Bulgaria. PeerJ 2016; 4: e2788.

29 Piquet J, Chavaillon JM, David P, et al. High-risk patients following hospitalisation for an acute exacerbation of COPD. Eur Respir J 2013; 42: 946-955.

30 Fuhrman C, Moutengou E, Roche N, et al. Prognostic factors after hospitalization for COPD exacerbation. Rev Mal Respir 2017; 34: 1-18.

31 Johannesdottir SA, Christiansen CF, Johansen MB, et al. Hospitalization with acute exacerbation of chronic obstructive pulmonary disease and associated health resource utilization: a population-based Danish cohort study. J Med Econ 2013; 16: 897-906.

32 Celli BR. Predictors of mortality in COPD. Respir Med 2010; 104: 773-779.

33 Celli BR, Cote CG, Marin JM, et al. The body-mass index, airflow obstruction, dyspnea, and exercise capacity index in chronic obstructive pulmonary disease. N Engl J Med 2004; 350: 1005-1012.

34 Cote CG. Surrogates of mortality in chronic obstructive pulmonary disease. Am J Med 2006; 119: Suppl. 1, 54-62. 